\title{
Abordagem conservadora em restaurações posteriores com compósitos: acompanhamento clínico de 2 anos
}

\author{
Conservative approach in possible restoration with composites: clinical accompa- \\ niment of 2 years
}

\author{
Alana de Castro Pereira', Ândria Milano San Martins' ${ }^{1}$, Vitor Schweigert Bona' ${ }^{1}$, Guilherme Carpena Lopes², \\ Sylvio Monteiro Junior ${ }^{2}$
}

\begin{abstract}
Para citar este artículo: Pereira AC, San Martins AM, Bona VS, Lopes GC, Monteiro JS. Abordagem conservadora em restaurações posteriores com compósitos: acompanhamento clínico de 2 anos. Ustasalud 2018; 17: 67-74
\end{abstract}

Licencia Creative Commons

\section{(c) $(1)(5)$} lo tanto, los lectores pueden acceder libremente a los artículos en su formato .pdf, igualmente podrán descargarlos y difundirlos; sin embargo no podrán modificarlos o alterarlos, adicionalmente se debe reconocer la autoría de las personas que figuran en las publicaciones, pero estas no podrán ser comercializadas.

\section{RESUMO}

A substituição de restaurações defeituosas por novas vem sendo um dos principais motivos de procedimentos restauradores. Porém sabe-se que durante a remoção do material antigo há um progressivo aumento do tamanho da cavidade, fragilizando o remanescente dental sadio. A odontologia moderna e o advento dos protocolos adesivos permitem que o odontólogo opte por um plano de tratamento mais conservador. Como uma alternativa à substituição total da restauração pode-se realizar tanto o re-polimento superficial devolvendo lisura e brilho ou ainda o reparo restaurador, que com adição de compósito é possível reanatomizar e regularizar a cor de restaurações de resinas antigas, porém bem adaptadas. O presente artigo tem como objetivo fazer um relato de caso clínico de uma abordagem conservadora em restaurações posteriores com compósitos, demonstrando a técnica e o sucesso clínico após um acompanhamento de dois anos.

Palavras-chave: Odontologia restauradora, Reparo restaurador, Reparo em resina composta.

\section{ABSTRACT}

Replacement of defective restorations with new ones has been a major reason for restorative procedures. However it is known that during the removal of the old material there is a progressive increase of the cavity size, weakening the healthy dental remnant. Modern dentistry and the advent of adhesive protocols allow the dentist to opt for a more conservative treatment plan. As an alternative to the total replacement of the restoration, both the surface re-polishing can be performed, restoring smoothness and gloss or restorative repair, which with the addition of composite can reanatomize and regularize the color of restorations of old, but well-adapted resins. The present article aims to make a clinical case report of a conservative approach in posterior composite restorations, demonstrating the technique and clinical success after a two - year follow - up.

Keywords: Restorative dentistry, Restorative repair, Composite resin repair.

\author{
'Mestre e Doutorando(a) em \\ Odontologia com ênfase em \\ Dentística pela Universidade Fe- \\ deral de Santa Catarina, Brasil \\ ${ }^{2}$ Professor titular do Programa de \\ Pós-graduação em Odontologia \\ da Universidade Federal de Santa \\ Catarina, Brasil \\ Autor responsável por \\ correspondência: \\ Alana de Castro Pereira \\ Email: \\ alanaxpereira@hotmail.com
}

12 de noviembre 2018. 


\section{INTRODUÇÃO}

Toda substituição de restauração requer o aumento do tamanho da cavidade muitas vezes às expensas dos tecidos dentais sadios. Brantley et al (1995) afirmaram que substituições de restaurações antigas levam a cavidades progressivamente maiores ${ }^{1}$.

Por muitos anos, a doença cárie era o motivo da realização de restaurações e atualmente a substituição de uma restauração por outra vem sendo uma das principais razões para confecção de novas restaurações diretas. Dentre alguns fatores inerentes ao material, o manchamento superficial se destaca como uma das causas de troca. Muitas vezes esse manchamento pode ser facilmente resolvido com soluções mais conservadoras, como o re-polimento da superfície ${ }^{2}$. Além disso com o advento odontologia adesiva permitiu-se também a reparação de restaurações pré-existentes, em vez da sua substituição completa, preservando a estrutura dental sadia que estaria em risco durante a remoção da restauração ${ }^{3}$.

Se tratando de uma abordagem minimamente invasiva, o reparo restaurador consiste na remoção parcial da resina antiga com adição de material resinoso corrigindo forma, anatomia e contorno. Geralmente são indicados nos casos onde as falhas das restaurações se restringem a pequenos defeitos de forma e cor $^{4,5}$. Quando acompanhado de um correto diagnóstico e bom planejamento, tende a preservar as estruturas dentais. A substituição da restauração seria indicada em casos de fratura, profundo manchamento marginal e diagnóstico de cárie secundária ${ }^{6}$.

Um dos principais objetivos da odontologia restauradora moderna é aumentar a longevidade das restaurações e preservar tecido dental sadio. Esse quesito pode, no entanto, depender de múltiplos fatores, como o operador, materiais, técnica operatória selecionada, localização e o tamanho da restauração, o risco e controle da doença cárie, bem como os hábitos parafuncionais ${ }^{7}$.

A durabilidade das restaurações dentárias está diretamente ligada à sua resistência à biodegradação pelos compostos salivares. A exposição dos materiais restauradores à base de resinas às condições adversas do ambiente bucal pode resultar em uma redução nas propriedades físico-mecânicas ${ }^{8}$.

A avaliação da qualidade de uma restauração deve basear-se em critérios clínicos detalhados para distinguir falhas que podem ser reparadas das que devem ser substituídas. Para tanto, a opinião pessoal do clínico pode não ser a forma mais segura de decisão. Em 1971 foram desenvolvidos os critérios USPHS (United States Public Health Service) por Cvar e Ryge, que são um conjunto de critérios clínicos utilizados para detectar a deterioração precoce e sinal de falhas em restaurações. Posteriormente sofreram alterações, sendo denominados critérios USPHS modificados. Porém foram desenvolvidos quando as amálgamas eram comumente usadas e os materiais adesivos tinham uma longevidade limitada9 .

$\mathrm{Na}$ atualidade foi necessária uma escala mais sensível e discriminativa do que os "critérios de Ryge modificados" e, em 2007, Hickel et al. propuseram um novo sistema baseado em três categorias: parâmetros estéticos (quatro critérios), parâmetros funcionais (seis critérios) e parâmetros biológicos (seis critérios). Cada critério pode ser expresso com cinco pontuações, três para aceitável e duas para não aceitável (uma para reparável e outra para substituição) permitindo uma descrição mais detalhada, realizando a avaliação em cinco passos como descritos na Tabela 1. O escore final em cada categoria é considerado o mais grave obtido entre todas as subcategorias. Os critérios definidos por Hickel et al. foram aprovados pela FDI World Dental Federation e considerado em 2008 como "Critérios Padrão". Com as primeiras utilizações, os feedbacks levaram a modificações de alguns critérios e pontuações em 2010. Foi observado que a classificação de cinco etapas também pode ser reduzida a quatro etapas (duas aceitáveis e duas inaceitáveis) ou a duas etapas combinando as pontuações 1-3 e as pontuações 4 e 5 em "restauração aceitável" e "restauração inaceitável”, respectivamente. Desde então, nenhuma atualização foi publicada sobre sua aplicação em estudos clínicos ${ }^{5-6,9}$.

Os critérios FDI, são práticos, relevantes e padronizados quando comparados com os USPHS. Possibilitando solucionar livremente, em virtude da grande variedade de classificações, além de serem mais sensíveis e apropriados aos materiais restauradores atuais e 
ao design de estudos clínicos. Por serem padronizados, facilitam as comparações entre as investigações ${ }^{4,10}$.

Tabela 1. Critérios FDI

\begin{tabular}{lc}
\hline Avaliação 5 passos & Avaliação 2 passos \\
\hline Clinicamente excelente/ Muito bom & Aceitável \\
\hline Clinicamente bom (após a correção muito bom) & \\
Clinicamente suficiente/ satisfatório (pequenas & \\
deficiências com efeitos adversos, mas ajustáveis & \\
sem danos permanentes aos dentes) & \\
$\begin{array}{l}\text { Clinicamente insatisfatório (reparações por } \\
\text { razões preventivas) }\end{array}$ & Inaceitável \\
\hline Pouco satisfatório (substituição necessária) & \\
\hline
\end{tabular}

O presente relato de caso tem como objetivo demonstrar que toda reintervenção em restaurações com compósitos precisa ser planejada e avaliada através de critérios que possam identificar os reais motivos da sua troca, uma vez que, em muitas situações pode-se optar por tratamentos mais conservadores alternativos à substituição, contribuindo com a longevidade das estruturas dentais sadias remanescentes.

\section{SEQUÊNCIA CLÍNICA}

Paciente, de 23 anos de idade, do sexo masculino, procurou atendimento na clínica da pós-graduação em odontologia na Universidade Federal de Santa Catarina situada no Brasil queixando-se de insatisfação estética e desconforto causado pela falta de contorno anatômico e perda da lisura superficial nas restaurações em resina composta que possuía nos dentes posteriores. Durante a anamnese o paciente relatou que os procedimentos foram realizados há aproximadamente sete anos. Ao exame clínico observou-se restaurações em resina composta do tipo Classe I nos dentes 16, 17, 26, 27 (Figura 1) e 36, 37, 46 e 47 (Figura 2).
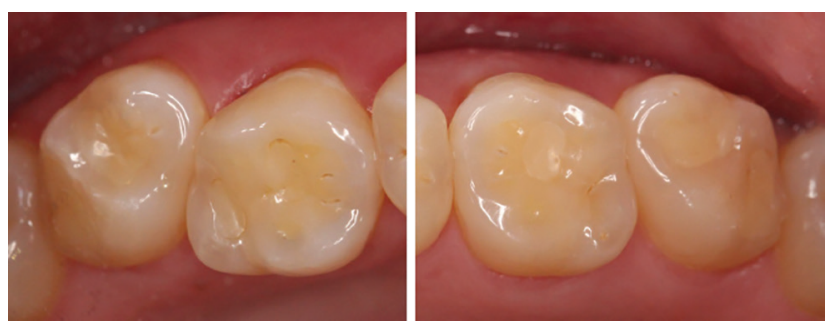

Figura 1. Restauração em resina composta tipo classe I nos dentes 16,17 e $26,27$.
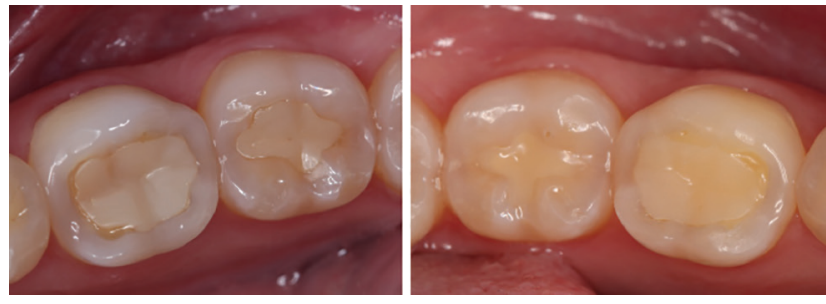

Figura 2. Restauração em resina composta tipo classe I nos dentes 46, 47 e 36, 37.

Ao exame radiográfico não foram observadas imagens radiolúcidas sugestivas de cárie nos dentes 16,17 , 46, 47 e 26, 27, 36, 37 (Figura 3). Notou-se no dente 46 uma imagem compatível com o que seria um tratamento expectante, confirmado com os relatos colhidos durante a anamnese.

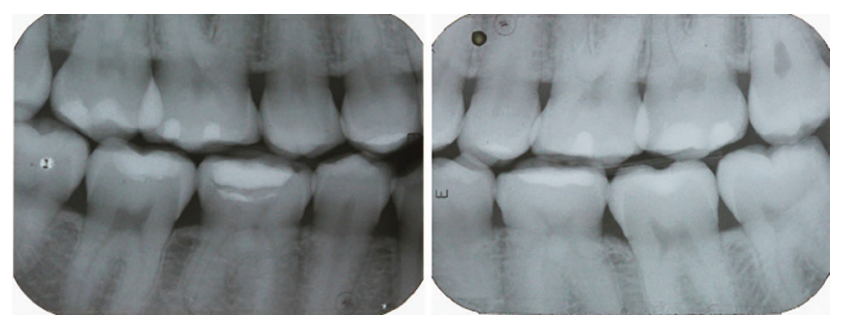

Figura 3. Radiografias interproximais de molares direito e esquerdo.

Após a avaliação clínica e radiográfica as restaurações foram classificadas segundos os critérios FDI para que fosse definido o plano de tratamento (Tabela 2). O planejamento consistiu em realizar apenas um polimento adicional nos dentes 16, 17, 26 e 27 que foram classificadas como "aceitáveis", para devolver o brilho e a lisura superficial; estabeleceu-se os procedimentos de reparo restaurador nos dentes 37 e 47 classificadas como "inaceitáveis" porém passíveis de reparações para reestabelecer a anatomia; e nos dentes 36 e 46 que foram classificadas como "inaceitáveis" ou pouco satisfatórias optou-se pela substituição completa da restauração antiga.

Os procedimentos restauradores foram realizados sob isolamento absoluto com dique de borracha (MADEITEX • São José dos Campos - São Paulo Brasil) e seguindo os critérios de assepsia do campo operatório. O preparo foi feito com pontas esféricas diamantadas (KG Sorensen, Barueri, SP, Brasil) em 
Tabela 2. Classificação dos dentes 16, 17, 26, 27, 36, 37, 46 E 47 segundo os critérios FDI

\begin{tabular}{|c|c|c|c|c|c|c|c|c|c|}
\hline Critérios FDI - 5 passos & DENTES & 16 & 17 & 26 & 27 & 36 & 37 & 46 & 47 \\
\hline Adaptação Marginal & & 3 & 3 & 3 & 1 & 5 & 4 & 5 & 4 \\
\hline Coloração & & 3 & 3 & 3 & 1 & 5 & 4 & 5 & 4 \\
\hline Fratura do Material/Retenção & & 1 & 1 & 1 & 1 & 5 & 3 & 5 & 3 \\
\hline Cárie secundária & & 1 & 1 & 1 & 1 & 3 & 1 & 3 & 1 \\
\hline Sensibilidade pós-operatória & & 1 & 1 & 1 & 1 & 5 & 1 & 5 & 1 \\
\hline Brilho superficial & & 3 & 3 & 3 & 3 & 5 & 4 & 5 & 4 \\
\hline Critérios FDI - 2 passos & & A & A & A & A & I & I & I & I \\
\hline
\end{tabular}

alta rotação compatíveis com o tamanho da cavidade. Nota-se que nas unidades 37 e 47 a instrumentação da cavidade teve a finalidade de remover parcialmente a restauração e promover uma asperização na superfície da resina antiga com o intuito de melhorar a adesão ao material novo. Já nas unidades 36 e 46 a remoção do material restaurador antigo foi praticamente completa, como pode ser observado na Figura 4. Verificou-se na parede pulpar da unidade 46 tecido dentinário reacional de um possível capeamento pulpar direto realizado há alguns anos, relatado na anamnese, e como medida de proteção aplicou-se cimento de hidróxido de cálcio (Dycal ${ }^{\oplus}$, Dentsply, Petrópolis, Rio de Janeiro, Brasil) antes dos procedimentos de adesão.
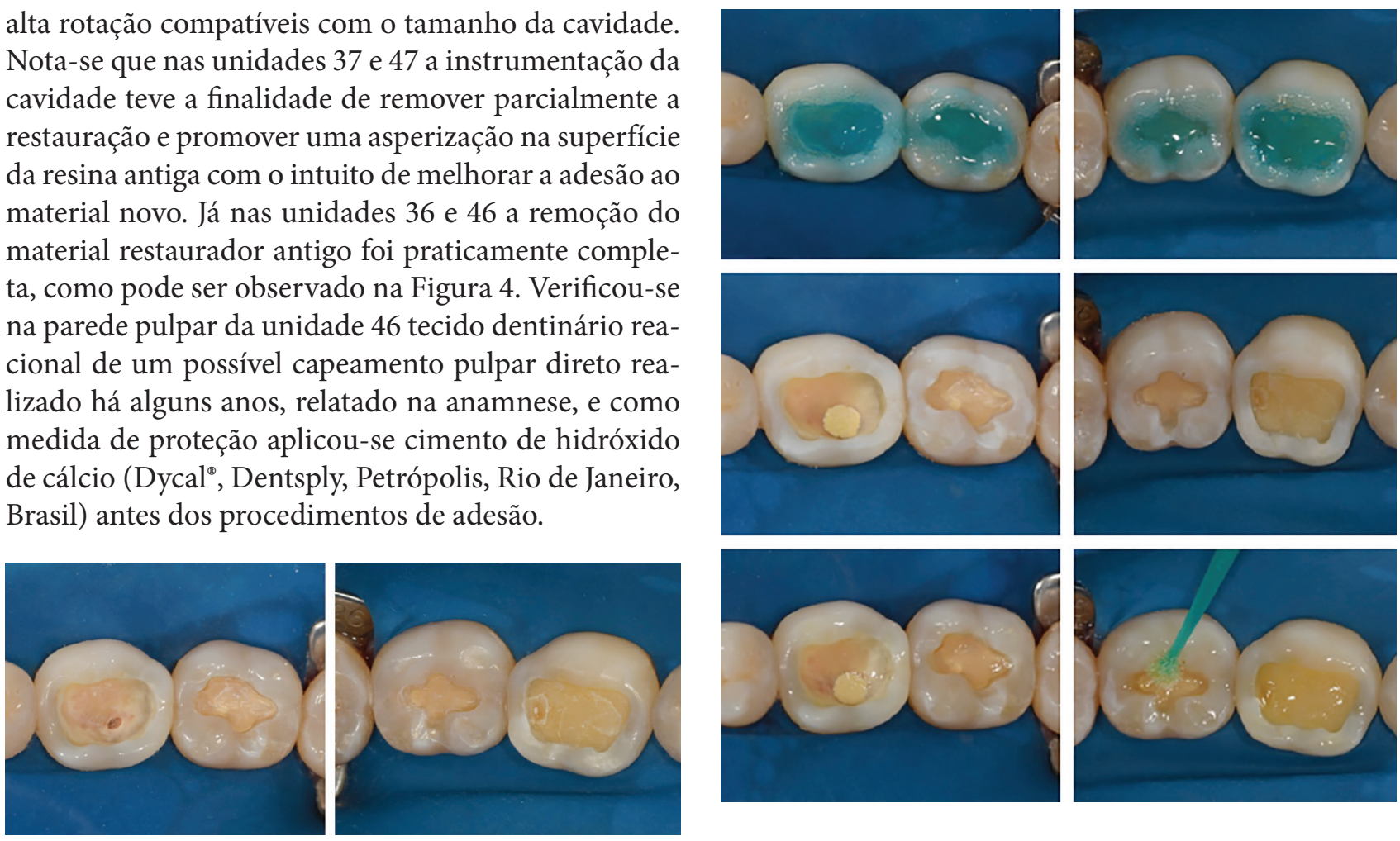

Figura 4. Preparo cavitário realizado sob isolamento absoluto.

Em todas as cavidades, mesmo aquelas para confecção do reparo, foram realizados os procedimentos adesivos que envolveram as etapas de condicionamento com ácido fosfórico a 37\% (Condac 37\% FGM, Joinville, SC, Brasil), lavagem das cavidades com spray de ar/água, secagem com proteção da dentina, aplicação do adesivo (Âmbar FGM, Joinville, SC, Brasil) e fotopolimerização, seguindo as recomendações do fabricante. A sequência clínica é ilustrada pela Figura 5.

Figura 5. Sequência clínica dos procedimentos adesivos nos dentes 46,47 e $36,37$.

A técnica restauradora eleita foi a da estratificação incremental utilizando resinas nanohíbridas (IPS Empress Direct, Ivoclar Vivadent, Schaan, Lietchtenstein) de esmalte e dentina, além de pigmentos resinosos (Tetric Color, Ivoclar Vivadent, Schaan, Liechtenstein). Primeiramente foram levados incrementos de dentina DA2 (cor compatível com a aferida antes do isolamento absoluto) com o auxílio de espátulas para resina composta. Cada incremento foi acomodado seguindo a anatomia e orientação das cúspides e foto- 
polimerizado individualmente por 10 segundos com uma intensidade de luz de $1.200 \mathrm{~mW} / \mathrm{cm}^{2}$ (Radii Cal, SDI, Bayswater, Austrália). Em seguida foram aplicadas pequenas quantidades de corante de cor Ocre na região dos sulcos principais e de cor White nas pontas de cúspides com o auxílio de pincéis delicados. Esta caracterização teve a finalidade de reproduzir a anatomia encontrada nos dentes hígidos vizinhos, promovendo naturalidade. Após a fotopolimerização dos corantes resinosos, a anatomia oclusal foi atentamente esculpida com os incrementos de resina de esmalte na cor EA2. Espátulas delgadas, sonda exploradora e pincéis de pelo de marta foram decisivos para a devolução dos contornos adequados das cúspides, sulcos principais e secundários. A sequência clínica é ilustrada pela Figura 6. Cada incremento de esmalte foi fotopolimerizado individualmente por 10 segundos e por fim aplicou-se uma pequena camada de gel hidrossolúvel bloqueador de oxigênio (Power Block, BM4, Palhoça, Santa Catarina, Brasil) para a fotopolimerização final por 60 segundos.
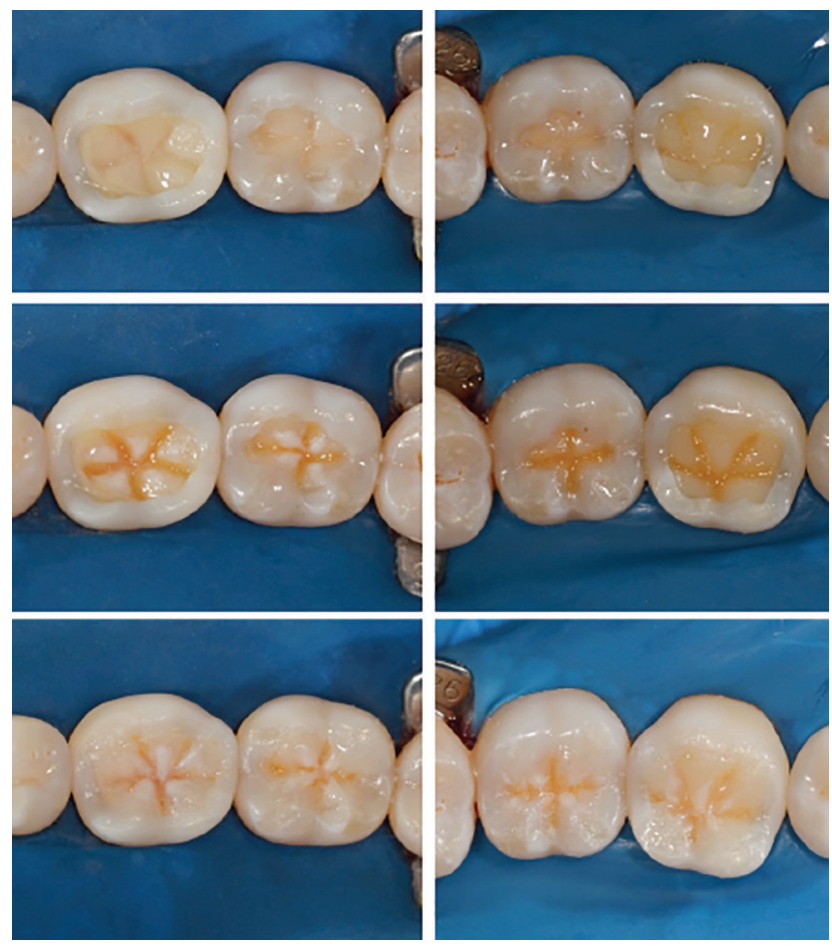

Figura 6. Técnica restauradora de estratificação incremental nos dentes 46, 47 e 36, 37. Inserção dos incrementos de dentina, seguido da aplicação de corantes e finalização com os incrementos de esmalte conferindo a anatomia oclusal.

Imediatamente após a remoção do isolamento absoluto checou-se os contatos com uma pinça de Müller e papel carbono (AccuFilm, Parkell in., Farmingdale, New York, EUA). Foram realizados, então, os ajustes oclusais e remoção dos excessos grosseiros com pontas diamantadas da série F e FF (KG Sorensen, Barueri, SP, Brasil). Aguardou-se um período de 48 horas para a realização dos procedimentos de acabamento e polimento, que por sua vez seguiu uma sequência decrescente de abrasividade com o kit de borrachas polidoras contendo óxido de alumínio em sua superfície (Astropol, Ivoclar Vivadent, Schaan, Liechtenstein). Finalizando o polimento, foi utilizado uma escova impregnada com carbeto de silício (Astrobrush, Ivoclar Vivadent, Schaan, Liechtenstein) que conferiu um aspecto espelhado à restauração ao final do processo. O paciente foi informado quantos aos cuidados com a higiene oral e liberado em seguida, satisfeito com o tratamento realizado. As figuras 7 e 8 ilustram a comparação das situações clínicas inicial e final, onde os contornos e anatomia característica oclusal foram devolvidos tanto nas cavidades em que houve a substituição completa (46 e 36), como naquelas em foi preconizado somente o reparo (47 e 37).
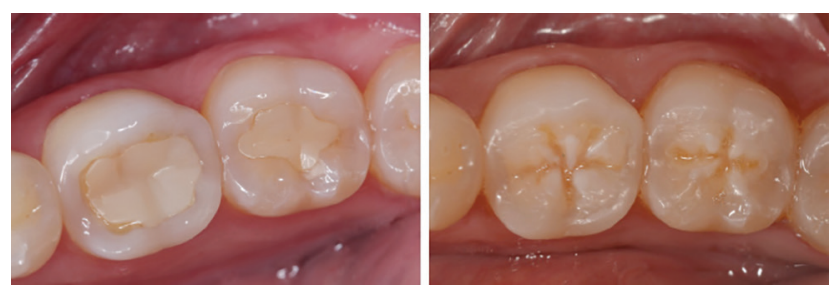

Figura 7. Situação clínica inicial e final nos dentes 46 e 47.
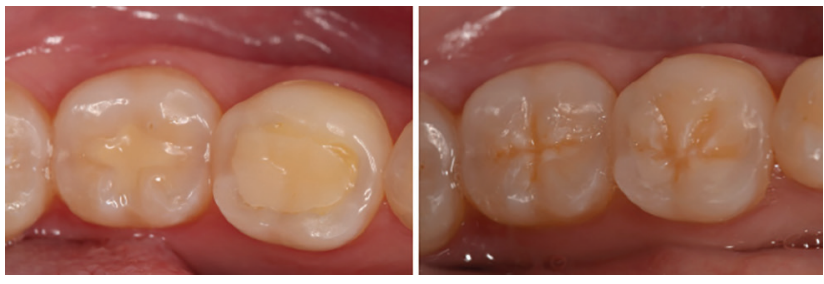

Figura 8. Situação clínica inicial e final nos dentes 36 e 37.

Após um período de 2 anos, o paciente retornou para consulta de rotina e ao exame clínico observou-se que as restaurações se encontravam bem adaptadas, sem manchamento marginal, alteração de cor ou fraturas. Verificou-se apenas uma leve perda de brilho superficial. O paciente não relatou nenhuma intercorrência ao longo desse período, tampouco episódios de sensibilidade pós-operatória. Figura 9 ilustra o as- 
pecto das restaurações classe I nas unidades $46,47,36$ e 37 após um acompanhamento clínico de dois anos. Os dentes submetidos ao reparo (47 e 37) apresentaram o mesmo sucesso clínico quando comparadas às unidades que foram totalmente substituídas (46 e 36).
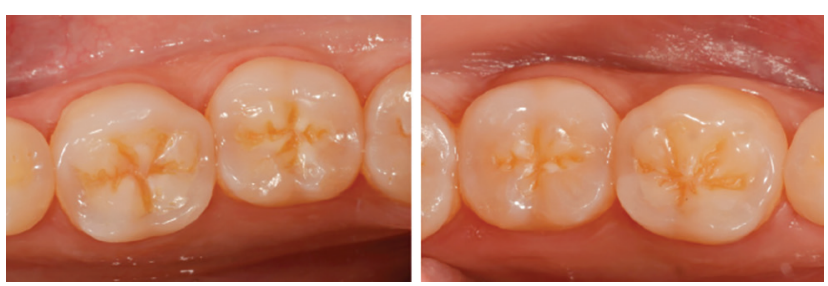

Figura 9. Restaurações em resina classe I nos dentes 46, 47 e 36, 37 no acompanhamento clínico de dois anos, aparentando sucesso clínico.

\section{DISCUSSÃO}

A decisão de substituir uma restauração antiga deve ir além de considerações pessoais do clínico. Brantley et al (1995) realizaram um estudo envolvendo 1.337 decisões para substituir restaurações existentes em dentes posteriores, e foi observado que 70 por cento de todas as recomendações resultaram em um aumento do número de superfícies restauradas. Eles denominaram como "ciclo de re-restauração" a prática comum que os dentistas vêm apresentando de substituírem completamente restaurações antigas indiscriminadamente. É importante salientar que esse ciclo resulta em dentes com cavidades e restaurações progressivamente maiores, o que pode trazer consequências para a sua longevidade1.

Contribuindo para a longevidade da estrutura dental, o planejamento para o caso determinou que as restaurações classe I nas unidades 16, 17, 26 e 27 fossem mantidas mesmo sendo observadas uma superfície de resina sem brilho e discreto manchamento marginal. Costa et al (2010) afirmaram em seu estudo que a rugosidade e o brilho da superfície dependem do tipo de resina composta e do sistema de polimento. Além disso, o efeito da escovação ao longo do tempo, por um efeito de abrasão, pode interferir na rugosidade superficial e diminuir o brilho, afetando assim a qualidade estética de uma restauração. Para o caso clínico descrito a solução abordada foi um tratamento conservador baseado no re-polimento dessas restaurações, removendo as manchas superficiais e devol- vendo lisura e brilho à restauração sem a necessidade de troca ou reparo. Essa proposta de tratamento concorda com o estudo realizado por Anfe (2009) onde foi observado que um desgaste de apenas 20 micrômetros $(\mu)$ foi suficiente para remoção de manchamento superficial provocados por café e vinho tinto em cinco tipos de resina composta avaliadas ${ }^{2,11}$.

Com base nos critérios FDI optou-se pelo reparo das restaurações em resina composta do tipo classe I nas unidades 37 e 47, onde o paciente relatava desconforto por conta de uma falta de anatomia, porém a adesão do material restaurador às estruturas dentais não estava comprometida. Masioli et al (2006) afirmaram que o procedimento de reparo é simples e eficaz na economia de tempo e estrutura dentária, e independe do uso de agentes de silanização, visto que a técnica utilizando apenas ácido fosfórico e sistema adesivo, também apresenta desempenho satisfatório. A mesma técnica foi realizada no presente caso clínico e acompanhada após 2 anos verificou-se sucesso clínico na estratégia escolhida ${ }^{4,11,12}$.

Sabe-se que em geral, as forças de união entre a resina de reparo são menores que as forças coesivas dos compósitos não reparados ${ }^{13}$, porém se as etapas dos procedimentos são bem realizadas e há uma união adequada entre a resina composta nova e a antiga, o reparo da restauração torna-se uma solução atrativa ${ }^{14}$.

Durante a confecção de uma restauração em resina composta a ligação molecular entre as camadas é alcançada devido a presença de uma camada inibida pelo oxigênio ${ }^{15-26}$. Como os reparos são confeccionados sobre resinas envelhecidas, não há a presença desta camada. Portanto diversas técnicas têm sido introduzidas e testadas para superar essa ausência e melhorar a adesão de reparos ${ }^{16}$. Dentre as técnicas disponíveis estão a asperização superficial com pontas diamantadas ${ }^{17}$, jateamento com oxido de alumínio $^{18,12,19,20}$, aplicação de silano ${ }^{18-20,21-24,}$ aplicação de adesivo $^{16-20,21-24,}$ aplicação de acetona ${ }^{21}$, ácido fosfóri$\mathrm{CO}^{17,19,24-26}$, e técnicas combinadas. Porém ainda não existe um consenso na literatura sobre a melhor técnica a ser empregada.

As restaurações em resina composta são altamente desafiadas no meio bucal e podem sofrer degradação ao longo do tempo ${ }^{3,9}$ e esse processo pode afetar di- 
retamente as propriedades superficiais como dureza e resistência ao desgaste. A exposição às condições adversas do ambiente bucal exige que esses materiais apresentem durabilidade significativa. Uma das propriedades mais importantes que determinam a durabilidade das restaurações dentárias é a sua resistência à biodegradação $0^{9,27,28}$. No presente caso clínico as restaurações foram reavaliadas em um acompanhamento de dois anos e verificou-se apenas uma pequena perda de brilho superficial. Isso mostra que ao longo do tempo pode-se fazer necessária a realização de procedimentos polidores para devolver lisura e brilho superficial.

\section{CONSIDERAÇÕES FINAIS}

A odontologia restauradora moderna deve prezar por procedimentos minimamente invasivos. O re-polimento periódico, bem como o reparo restaurador são opções alternativas de tratamento conservador que garantem não só a preservação de remanescente dental sadio como sucesso clínico restaurador.

\section{REFERÊNCIAS}

1. Brantley CF, Bader JD, Shugars DA, Nesbit SP. Does the cycle of rerestoration lead to larger restorations? J Am Dent Assoc. 1995;126(10):1407-13.

2. Anfe TEA. Avaliação da possiibilidade de remoção do manchamento de resinas compostas submetidas ao envelhecimento artificial através do re-polimento. [Dissertação de Mestrado]. São Paulo. Faculdade de Odontologia da USP; 2009.

3. Rodrigues SA Jr, Ferracane JL, Della Bona A. Influence of surface treatments on the bond strength of repaired resin composite restorative materials. Dent Mater. 2009;25(4):442-51. DOI: 10.1016/j.dental.2008.09.009.

4. Hickel R, Peschke A, Tyas M, Mjör I, Bayne S, Peters M, Hiller KA, Randall R, Vanherle G, Heintze SD. FDI World Dental Federation: clinical criteria for the evaluation of direct and indirect restorations-update and clinical examples. Clin Oral Investig. 2010;14(4):349-66. DOI: 10.1007/s00784-010-0432-8.

5. Hickel R, Roulet JF, Bayne S, Heintze SD, Mjör IA, Peters M, Rousson V, Randall R, Schmalz G, Tyas M, Vanherle G. Recommendations for conducting controlled clinical studies of dental restorative materials. Clin Oral Inves- tig. 2007;11(1):5-33. Erratum in: Clin Oral Investig. 2008 Mar;12(1):97. PubMed PMID: 17262225.

6. Ribeiro MDF \& Pazinatto FB. Critérios clínicos para decisão entre substituição ou reparo de restaurações em resina composta - revisão de litaratura. Rev. bras. odontol., Rio de Janeiro. 2016;73(3):223-30.

7. Pallesen U, van Dijken JW. A randomized controlled 30 years follow up of three conventional resin composites in Class II restorations. Dent Mater. 2015;31(10):1232-44.

8. Bagheri R, Tyas MJ, Burrow MF. Subsurface degradation of resin-base composites. Dent Mater. 2007 Aug;23(8):944-51. PMID: 16996586.

9. Cvar JF, Ryge G. Reprint of criteria for the clinical evaluation of dental restorative materials. 1971. Clin Oral Investig. 2005;9(4):215-32.

10. Marquillier T, Doméjean S, Le Clerc J, Chemla F, Gritsch K, Maurin JC, Millet P, Pérard M, Grosgogeat B, Dursun E. The use of FDI criteria in clinical trials on direct dental restorations: A scoping review. J Dent. 2018; 68:1-9. DOI: 10.1016/j.jdent.2017.10.007. PMID: 29055692.

11. Costa J, Adams-Belusko A, Riley K, Ferracane JL. The effect of various dentifrices on surface roughness and gloss of resin composites. J Dent. 2010;38 Suppl 2:e123-8.

12. Masioli et al. Reparo em Restaurações de Resina Composta: procedimento simples e conservador. UFES Rev. Odontol., Vitória. 2006; 8(3):38-43.

13. Azarbal P, Boyer DB, Chan KC. The effect of bonding agents on the interfacial bond strength of repaired composites. Dent Mater. 1986;2(4):153-5. PMID: 2943623.

14. Bacchi A, Assad-Calvante LM, Jochims-Schneider LF, Xediek-Consani RL. Reparos em restaurações de resina composta: revisão de literatura. RFO UPF. 2010;15(3):331-35.

15. Li, J. Effects of surface properties on bond strength between layers of newly cured dental composites. Journal of oral rehabilitation. 1997;24(5):358-60.

16. Tezvergil A, Lassila LVJ, Vallittu PK. Composite-composite repair bond strength: effect of different adhesion primers. Journal of dentistry. 2003;31(8):521-5.

17. Bonstein, Garlapo D, Donarummo J, Bush PJ. Evaluation of varied repair protocols applied to aged composite resin. J Adhes Dent. 2005;7(1):41-9.

18. Bacchi A, Consani RL, Sinhoreti MA, Feitosa VP, Cavalcante LM, Pfeifer CS, et al. Repair bond strength in aged 
methacrylate- and silorane-based composites. J Adhes. 2013;15(5):447-52.

19. Ozcan M, Valandro LF, Amaral R, Leite F, Bottino MA. Bond strength durability of a resin composite on a reinforced ceramic using various repair systems. Dent Mater. 2009;25(12):1477-83. DOI: 10.1016/j.dental.2009.06.020.

20. Özcan M, Corazza PH, Marocho SM, Barbosa SH, Bottino MA. Repair bond strength of microhybrid, nanohybrid and nanofilled resin composites: effect of substrate resin type, surface conditioning and ageing. Clin Oral Investig. 2013;17(7):1751-8. DOI: 10.1007/s00784-012-0863-5.

21. Hamano N, Chiang YC, Nyamaa I, Yamaguchi H, Ino S, Hickel R, et al. Effect of different surface treatments on the repair strength of a nanofilled resin-based composite. Dent Mater J. 2011;30(4):537-45.

22. Hamano N, Chiang YC, Nyamaa I, Yamaguchi H, Ino S, Hickel R et al. Repair of silorane-based dental composites: influence of surface treatments. Dent Mater. 2012;28(8):894-902. DOI: 10.1016/j.dental.2012.04.014.

23. Lührs AK, Görmann B, Jacker-Guhr S, Geurtsen W. Repairability of dental siloranes in vitro. Dent Mater. 2011;27(2):144-9. DOI: 10.1016/j.dental.2010.09.009.
24. El-Askary FS, Fawzy AS.; Abd Elmohsen HM. Tensile bond strength of immediately repaired anterior microfine hybrid restorative composite using nontrimmed hourglass specimens. 2009;11(1):41-7.

25. Loomans BA, Cardoso MV, Roeters FJ, Opdam NJ, De Munck J, Huysmans MC, Van Meerbeek B. Is there one optimal repair technique for all composites? Dent Mater. 2011;27(7):701-9. DOI: 10.1016/j.dental.2011.03.013.

26. Brosh T, Pilo R, Bichacho N, Blutstein R. Effect of combinations of surface treatments and bonding agents on the bond strength of repaired composites. J Prosthet Dent. 1997;77(2):122-6. PMID: 9051597.

27. Jaffer F, Finer Y, Santerre JP. Interactions between resin monomers and commercial composite resins with human saliva derived esterases. Biomaterials. 2002;23(7):170719.PMID: 11922474.

28. Costa TR, Serrano AM, Atman AP, Loguercio AD, Reis A. Durability of composite repair using different surface treatments. J Dent. 2012;40(6):513-21. DOI: 10.1016/j. jdent.2012.03.001. 